Revista de Economia Política, vol. 29, $n^{\circ} 3$ (115), pp. 213-231, julho-setembro/2009

\title{
Qual a sensibilidade dos investimentos privados a aumentos na carga tributária brasileira? Uma investigação econométrica
}

\author{
CLÁUDIO H. DOS SANTOS \\ MANOEL CARLOS DE CASTRO PIRES*
}

How does Brazilian private investment respond to increases in the gross tax burden? An econometric investigation. This article aims to offer three contributions to the debate on the recent performance of the Brazilian economy. First, it presents approximate quarterly figures for the Brazilian private investment beginning in 1995 . Second, it presents estimates of the Brazilian "investment function" which are robust to the presence of structural breaks. Third, it discusses the sensitivity of private investment to increases in the gross aggregate tax rate. Estimates based on national accounting data prior to march 2007 seem to indicate that an increase of $1 \%$ of GDP in the tax rate is associated with a reduction of $1 \%$ in private investment.

Keywords: Brazilian private investment; Brazilian aggregate tax rate; Cointegration analysis with structural breaks.

JEL Classification: C22; C82; E22.

\section{INTRODUÇÃO}

Os últimos 12 anos testemunharam uma elevação da carga tributária bruta brasileira da ordem de nove pontos percentuais do PIB, fenômeno esse citado por

\footnotetext{
* Cláudio H. dos Santos é técnico de pesquisa e planejamento e coordenador de finanças públicas do IPEA e pesquisador associado do Levy Economics Institute. E-mail: claudio.santos@ipea.gov.br Manoel Carlos de Castro Pires é técnico de pesquisa e planejamento do IPEA e coordenador geral de política fiscal da Secretaria de Política Econômica do Ministério da Fazenda. E-mail: manoel.pires@ipea. gov.br. Os autores agradecem a Alexandre Manoel da Silva, Alexandre Ywata de Carvalho, Bruno Cruz, Jorge Abraão de Castro, José Celso Cardoso Jr., Márcio Ribeiro, Mérida Medina, Thiago Velloso, Waldery Rodrigues Jr. e a dois pareceristas anônimos pelas contribuições. Os erros remanescentes são de exclusiva responsabilidade dos autores.
} 
muitos macroeconomistas (e.g., Pastore e Pinotti, 2006; Giambiagi.,2006; Velloso, 2006 , entre outros) como uma das causas das baixas taxas de investimento e crescimento do período.

O argumento desses autores é simples: elevações na carga tributária desincentivariam os investimentos privados e, por essa via, reduziriam o crescimento do produto potencial do país. Portanto, maiores taxas de crescimento seriam alcançadas se cortes significativos fossem efetuados tanto nos gastos públicos correntes como na carga tributária bruta da economia - dada a manutenção de uma meta de superávit primário compatível com a redução gradual da dívida líquida do setor público como percentagem do PIB (DLSP) ${ }^{1}$. Em resumo, tanto o "aprofundamento do ajuste fiscal" (i.e., a diminuição contínua da DLSP) como a "melhoria da qualidade" do mesmo (i.e., a diminuição conjunta da carga tributária bruta e dos gastos correntes) seriam pre-condições para a aceleração do crescimento brasileiro.

Implícitas no discurso acima estão as hipóteses de que a sensibilidade do investimento privado brasileiro a diminuições na carga tributária bruta e na taxa básica de juros da economia são relativamente altas. Com efeito, se essas sensibilidades forem baixas, o aumento no crescimento do produto potencial (i.e., o "benefício social”) associado a tais medidas também será baixo. Por outro lado, poucos discordariam que o "custo social" de uma redução significativa da carga tributária e dos gastos públicos correntes é alto. Há, de fato, poucas dúvidas que (i) o aumento da carga tributária nos últimos anos tem sido destinado a financiar a elevação dos gastos públicos com previdência e assistência social (Giambiagi, 2006); e (ii) que esses gastos têm contribuído para a redução da desigualdade de renda no país (Soares, 2006). Percebe-se, assim, que o atual debate sobre a política fiscal brasileira é semelhante ao gerado pela "teoria do bolo" atribuída ao ex-ministro Delfim Netto (1973).

Esse texto visa contribuir para o aprofundamento do debate sobre os impactos macroeconômicos da tributação no Brasil - e dos termos do trade-off entre tributação/distribuição e crescimento à disposição dos brasileiros - de três maneiras. Primeiramente, na primeira seção, propomos uma tipologia para "mapear" a literatura econométrica recente sobre os determinantes do investimento no Brasil e discutimos algumas características centrais dessa última. Na segunda seção, fazemos considerações sobre os dados "de investimento" brasileiros e apresentamos uma série trimestral para o investimento privado baseada em dados das contas nacionais trimestrais (anteriores à revisão de março de 2007), das "despesas de investimento" públicas (compilados pelo Ministério do Planejamento e pela Secretaria de Política Econômica, SPE) e no estudo de Gobetti (2006). Na terceira seção utilizamos essa última série - além da carga tributária brasileira trimestral apresentada em Dos Santos e Costa (2007) e outras variáveis mais tradicionais - para

\footnotetext{
${ }^{1}$ Que, por sua vez, contribuiria para o investimento e o crescimento ao permitir reduções graduais na taxa básica de juros da economia.
} 
obter estimativas econométricas dos determinantes do investimento privado brasileiro no período após o Plano Real, concluindo por um valor próximo a menos um para a "semielasticidade-carga tributária" do investimento privado.

\section{BREVES NOTAS SOBRE A LITERATURA ECONOMÉTRICA RECENTE ACERCA OS DETERMINANTES DO INVESTIMENTO PRIVADO NO BRASIL}

Ainda que assumindo o risco de simplificação excessiva, parece possível dividir a literatura econométrica sobre o investimento agregado brasileiro em dois grupos (ver Tabela 1), quais sejam, um composto por textos com "funções investimento" tradicionais e outro de cunho mais "clássico" e influenciado pelo artigo de Feldstein e Horioka (1980) - preocupado em estimar relações de cointegração entre os níveis de poupança interna e investimento doméstico.

\section{Tabela 1: Uma lista dos estudos econométricos recentes sobre o investimento privado brasileiro}

\begin{tabular}{|l|l|}
\hline Funções Investimento Independentes & Funções Poupança Independentes \\
\hline Rocha e Teixeira (1996) & Sachsida (1999) \\
\hline Melo e Rodrigues Jr. (1998) & Sachsida e Abi-Ramia (2000) \\
\hline Reis et al. (1999) & Sachsida e Mendonça (2006) \\
\hline Cruz e Teixeira (1999) & \\
\hline Pereira (2001)-dados trim. & \\
\hline Ribeiro e Teixeira (2001) & \\
\hline Cavalcanti et al. (2002)-dados trim. & \\
\hline Muinhos e Alves (2003)-dados trim. & \\
\hline
\end{tabular}

Fonte: Elaborado pelos autores.

Restringindo-nos ao primeiro grupo, notamos que as estimativas da "função investimento privado" brasileira são baseadas nas teorias neoclássico-keynesianas sobre os determinantes do investimento agregado e, portanto, supõem que essa última variável está correlacionada com (i) o PIB e/ou o grau de utilização da capacidade produtiva da economia (positivamente); (ii) a disponibilidade de crédito (positivamente); (iii) o "custo de utilização do capital" (negativamente); (iv) o grau de incerteza (positiva ou negativamente); e (v) o nível dos investimentos públicos (positiva ou negativamente).

Em segundo lugar, o fato das contas nacionais trimestrais do IBGE não desagregarem a formação bruta de capital fixo (doravante, FBCF) da economia em seus componentes público e privado tem se mostrado um obstáculo ao estudo dos determinantes do investimento privado no Brasil. Com efeito, a maioria dos artigos utiliza dados anuais (e, portanto, pequenas amostras) sobre a FBCF privada da economia. As exceções que confirmam a regra são Pereira (2001) - que utiliza 
os dados trimestrais do IBGE sobre a FBCF total da economia como proxy para a FBCF privada - e os trabalhos de Cavalcanti et al. (2002) e Muinhos e Alves (2003) que trabalham com dados trimestrais não especificados sobre o investimento público e a carga tributária. Os dois últimos artigos (e Reis et al., 1999) diferem dos demais ainda por (i) trabalharem com séries desagregadas do investimento em "máquinas e equipamentos" e em "construção civil"; e (ii) não estarem preocupados em "testar" a teoria " $A$ " ou "B" sobre o investimento, mas em apresentar uma equação para essa variável que se ajuste melhor aos dados.

Em terceiro lugar, esses estudos não incluem a carga tributária da economia entre os determinantes do investimento privado - ainda que Muinhos e Alves (2003) incluam as receitas do governo entre os determinantes da FBCF pública e que vários autores incluam a FBCF pública como determinante da FBCF privada. Com efeito, o "custo de utilização do capital" é usualmente aproximado nesses modelos pela taxa de juros real da economia e/ou alguma medida do "preço relativo dos bens de capital". Ainda que a exclusão da carga tributária possa fazer sentido em períodos nos quais ficou mais ou menos constante como percentagem do PIB (e.g., entre os anos 1970-1995), há poucos motivos para se achar que a significativa elevação da carga tributária entre os anos 1951-1970 e 1995-2006 tenha sido neutra do ponto de vista do investimento.

Em quarto lugar, pouca atenção é dada aos problemas associados à existência de quebras estruturais nas séries. Com efeito, ainda que alguns trabalhos (e.g., Pereira, 2001; Ribeiro e Teixeira, 2001) tomem o cuidado de aplicar testes de Perron (1989) para garantir que os resultados dos testes de raiz unitária não estejam contaminados pela existência de quebras estruturais, as implicações dessas últimas para os resultados dos testes de cointegração convencionais (e.g., Leybourne e Newbold, 2003; Hoglund e Ostermark, 2003; Noh e Kim, 2003) são ignoradas nessa literatura.

\section{MEDIDAS APROXIMADAS DO INVESTIMENTO PRIVADO TRIMESTRAL}

Recentemente, três desenvolvimentos contribuíram para atenuar o problema da falta de dados de alta frequência sobre o investimento privado e variáveis relacionadas no Brasil. Primeiramente, a Secretaria do Tesouro Nacional (STN) tem feito esforço no sentido de ampliar a base de dados sobre a execução orçamentária-financeira de estados e municípios. Em segundo lugar, o estudo de Gobetti (2006) iluminou a relação entre os dados das "despesas de investimento" da execução orçamentária da União/estados/municípios/estatais e os dados da "formação bruta de capital fixo" (da administração pública) das contas nacionais - além de apresentar estimativas dessa última variável para os anos de 2004 e 2005 . Finalmente, estimativas da carga tributária brasileira trimestral estão agora disponíveis (ver Dos Santos e Costa, 2007).

Os dois primeiros desenvolvimentos nos permitiram construir medidas trimestrais aproximadas para a FBCF privada brasileira a partir de 1995 (ver segun- 
da seção, enquanto o terceiro desenvolvimento nos permitiu estimar a sensibilidade de nossas medidas aproximadas a elevações na carga tributária bruta da economia após o Plano Real (terceira seção). A vantagem de trabalhar com dados trimestrais é a possibilidade de utilizar amostras maiores e, com isso, aumentar a qualidade dos resultados econométricos ${ }^{2}$.

\section{Alguns cuidados a tomar com os dados de investimento brasileiros}

Os dados brasileiros sobre investimentos têm pelo menos três limitações importantes. Em primeiro lugar, o IBGE não calcula deflatores trimestrais - as séries "encadeadas" publicadas nas contas nacionais trimestrais têm como referência os dados nominais do ano anterior - e esse fato dificulta a interpretação das medidas trimestrais "de volume" do IBGE. Analisando, por exemplo, os dados nominais e os índices de volume da FBCF publicados pelo IBGE para os quatro trimestres de 1995 (respectivamente, R \$ 31,5, R \$ 34,3, R\$ 33,51 e R \$ 33,46 bilhões e 121,8, $120,8,112,0$ e 109,3 , com média de $1990=100$ ) poder-se-ia supor que a variação do deflator do investimento foi de $2,4 \%$ no quarto trimestre desse $a^{3}{ }^{3}$. Entretanto, o que a queda do índice encadeado do IBGE de 112 para 109,3 traduz é que a composição dos investimentos no terceiro e no quarto trimestres de 1995 foi tal que os últimos foram $2,7 \%$ menores que os primeiros se ambos são medidos a preços médios de 1994.

Essa tecnicalidade é importante porque o fato de os preços médios de 1994 terem sido muito menores do que os verificados em 1995 (por conta da alta inflação nos dois primeiros trimestres de 1994) ajuda a explicar o fato do índice de volume da FBCF do IBGE ter caído de 125 para 109 entre o quarto trimestre de 1994 e o quarto trimestre de 1995, a despeito do aumento nominal de cerca de $15 \%$ na FBCF entre os períodos (contra uma inflação de cerca de $20 \%$ medida pelo IPCA). Felizmente, aproximações trimestrais do "deflator do investimento" podem ser construídas utilizando-se as variações de índices de preço de máquinas e equipamentos e materiais de construção ponderadas pelos pesos desses setores na FBCF anual da economia. De posse dessas aproximações podemos construir medidas "a preços constantes" dos investimentos públicos e privados a partir dos valores nominais dessas variáveis - contornando, assim, o viés apontado nos índices "de volume" do IBGE em 1995.

Em segundo lugar, o conceito de FBCF - a variável de investimento publicada trimestralmente pelo IBGE - é muito amplo, abarcando (i) investimentos familia-

\footnotetext{
${ }^{2}$ Simulações expostas em Patterson (2000) sugerem, por exemplo, que, a despeito da "superconsistência” dos estimadores de Engle e Granger (1987), as propriedades dos mesmos em amostras com menos de 40 observações não são boas. Note que a amostra utilizada nesse trabalho (47 observações) é maior que a da maioria dos estudos citados.

${ }^{3}$ Dado que o valor nominal da FBCF permaneceu essencialmente constante com relação ao terceiro trimestre e o índice de volume caiu de 112 para 109,3.
} 
res em residências; (ii) investimentos de empresas e/ou do governo em novas instalações administrativas e em infraestrutura e, (iii) investimentos de empresas em novas máquinas e equipamentos. Uma vez que esses tipos de investimentos tendem a ser determinados por variáveis bastante diferentes entre si, a tentativa de estimar uma "função investimento" que explique o agregado deles é, de saída, um exercício problemático.

Em terceiro lugar, julgamentos sobre a complementaridade ou não dos investimentos públicos e dos investimentos privados no Brasil, por exemplo, são complicados pelo fato dos dados trimestrais do IBGE não desagregarem a FBCF total da economia em seus componentes público e privado. Dados anuais da FBCF pública e privada estão disponíveis, mas mesmo esses devem ser vistos com cuidado, uma vez que o IBGE trata as empresas estatais como parte do setor privado ${ }^{4}$.

As implicações dessas limitações não devem ser subestimadas. Poder-se-ia conjeturar, por exemplo, que investimentos públicos em infraestrutura são complementares ao investimento privado em máquinas e equipamentos, enquanto investimentos públicos na produção de bens finais (como latas de óleo lubrificante para veículos, por exemplo) são substitutos dos investimentos privados. Uma vez que somente os dados agregados (tanto da FBCF pública como da privada) estão disponíveis, não temos como testar essas hipóteses.

\section{Construindo séries trimestrais da FBCF pública e da FBCF privada}

A metodologia que utilizamos para desagregar o dado da FBCF das contas nacionais trimestrais em seus componentes público e privado consistiu de duas etapas. Primeiramente, estimamos séries trimestrais da FBCF pública a partir de dados mensais compilados pela SPE sobre as "despesas de investimento" das três esferas de governo e das empresas estatais federais ${ }^{5}$ e de dados anuais da FBCF da administração pública e das empresas estatais publicados pelo IBGE. Em segundo lugar, calculamos a FBCF privada por resíduo, i.e., como o resultado da subtração da FBCF total trimestral da economia por nossas estimativas da FBCF pública trimestral. $\mathrm{O}$ restante dessa seção detalha os procedimentos adotados na construção da série da FBCF pública trimestral.

Notamos que, a despeito dos conceitos de "despesas de investimento" (da contabilidade pública) e de "FBCF" (do IBGE) serem diferentes (Gobetti, 2006, p. 34 e pp. 37-39), é possível utilizar o primeiro para aproximar o segundo. Com efeito, a Tabela 2 deixa claro que as aproximações anuais obtidas no caso de estados e municípios são bastante razoáveis, com o governo federal respondendo pela maior parte da imprecisão total. O motivo para essa discrepância é que os dados

\footnotetext{
${ }^{4}$ Isso dificulta a interpretação dos resultados obtidos na literatura. Por exemplo, enquanto Mello e Rodrigues (1998) e Cruz e Teixeira (1999) incluem os investimentos estatais na definição de "investimento público”, isso não ocorre nos estudos de Rocha e Teixeira (1996) e Ribeiro e Teixeira (2001).

${ }^{5}$ Obtivemos os dados através de comunicação pessoal com a SPE.
} 
das "despesas de investimento" incluem tanto a FBCF propriamente dita quanto as "transferências de capital" (que não são consideradas FBCF pelo IBGE e que são muito importantes no caso da União).

Tabela 2: Grau de Precisão (Anualizado) da FBCF pública dados da SPE (em R\$ bilhões correntes) ${ }^{6 *}$ estimado por Gobetti (2006).

\begin{tabular}{|c|c|c|c|c|c|c|c|c|c|}
\hline \multirow{2}{*}{$\begin{array}{l}\text { Dado/ } \\
\text { Ano }\end{array}$} & \multicolumn{2}{|c|}{$\begin{array}{l}\text { Investimentos da } \\
\text { União }\end{array}$} & \multicolumn{2}{|c|}{$\begin{array}{c}\text { Investimentos dos } \\
\text { Estados }\end{array}$} & \multicolumn{2}{|c|}{$\begin{array}{l}\text { Investimentos dos } \\
\text { Municípios }\end{array}$} & \multicolumn{2}{|c|}{$\begin{array}{l}\text { Investimento } \\
\text { Público Total }\end{array}$} & \multirow{2}{*}{$\begin{array}{c}\text { FBCF } \\
\text { Pública } \\
\text { CEI//BG }\end{array}$} \\
\hline & $\begin{array}{l}\text { Dados } \\
\text { IBGE** }^{*}\end{array}$ & $\begin{array}{l}\text { Dados } \\
\text { SPE }\end{array}$ & $\begin{array}{l}\text { Dados } \\
\text { IBGE* }\end{array}$ & $\begin{array}{l}\text { Dados } \\
\text { SPE }\end{array}$ & $\begin{array}{l}\text { Dados } \\
\text { IBGE* }^{*}\end{array}$ & $\begin{array}{l}\text { Dados } \\
\text { SPE }\end{array}$ & $\begin{array}{l}\text { Dados } \\
\text { IBGE* }^{*}\end{array}$ & $\begin{array}{l}\text { Dados } \\
\text { SPE }\end{array}$ & \\
\hline 1995 & 4,06 & 4,93 & 3,71 & 4,40 & 8,07 & 7,32 & 15,84 & 16,65 & 16,38 \\
\hline 1996 & 4,06 & 5,73 & 5,72 & 4,15 & 9,64 & 8,72 & 19,42 & 18,60 & 17,97 \\
\hline 1997 & 4,89 & 7,54 & 7,82 & 6,21 & 6,47 & 5,29 & 19,18 & 19,04 & 17,21 \\
\hline 1998 & 5,07 & 8,28 & 12,72 & 9,18 & 8,05 & 6,99 & 25,84 & 24,46 & 25,63 \\
\hline 1999 & 4,11 & 6,93 & 6,01 & 4,89 & 8,41 & 6,34 & 18,53 & 18,17 & 16,86 \\
\hline 2000 & 5,62 & 10,10 & 8,39 & 7,67 & 9,18 & 8,70 & 23,19 & 26,47 & 20,87 \\
\hline 2001 & 6,54 & 14,58 & 11,35 & 10,48 & 8,61 & 8,09 & 26,5 & 33,15 & 26,42 \\
\hline 2002 & 5,45 & 10,13 & 11,62 & 10,80 & 14,17 & 13,58 & 31,24 & 34,51 & 29,64 \\
\hline 2003 & 4,22 & 6,45 & 9,24 & 8,67 & 12,73 & 12,27 & 26,19 & 27,38 & 26,40 \\
\hline $2004^{*}$ & 7,19 & 10,87 & 12,36 & 13,99 & 16,23 & 12,89 & 35,78 & 37,74 & ND \\
\hline $2005^{*}$ & 11,28 & 17,14 & 15,91 & 18,34 & 13,74 & 10,77 & 40,93 & 46,25 & ND \\
\hline Total & 62,49 & 102,67 & 104,85 & 98,78 & 115,30 & 100,97 & 282,64 & 302,42 & ND \\
\hline
\end{tabular}

Fonte: Elaborado pelos autores com base nos dados primários do IBGE, SPE e Gobetti (2006).

* Estimado por Gobetti (2006).

Cumpre mencionar três pontos importantes sobre a Tabela 2. Primeiramente, os valores para 2004 e 2005 são estimativas construídas por Gobetti (2006), visto que as contas nacionais apresentam dados apenas até 2003. Em segundo lugar, as séries da SPE sobre o investimento dos estados e municípios apresentam quebras estruturais em 2004. Com efeito, enquanto a série municipal passa a refletir mais de perto a definição de FBCF nesse ano, o contrário ocorre com a série estadual. Uma vez que tais quebras se deram no nível e não no padrão sazonal das séries, entretanto, consideramos que nossas estimativas não seriam afetadas por elas (ver abaixo). Em terceiro lugar, os dados acima ignoram os problemas na contabilização dos gastos de investimento público da União apontados por Gobetti (2006, ver abaixo). Isso porque os dados do IBGE também ignoram esses problemas.

Tendo em vista os resultados acima, consideramos que "aproximações" da FBCF pública trimestral poderiam ser obtidas aplicando-se o padrão sazonal verificado na

\footnotetext{
${ }^{6}$ Uma vez que as contas nacionais não desagregam o investimento público por esfera do governo, os "dados do IBGE" citados na Tabela 3 foram obtidos, salvo menção em contrário, nas publicações "Regionalização de Transações do Setor Público" (para os anos 1995-2000) e "Finanças Públicas do Brasil” (para os anos 2001-2003).
} 
série de "despesas de investimento" aos dados anuais da FBCF pública publicados pelo IBGE. De fato, isso foi feito no caso da FBCF de estados, municípios e empresas estatais. A excepcionalidade do caso da FBCF da União é explicada abaixo.

O motivo de não termos aplicado a sazonalidade dos dados contábeis "de despesas de investimento" aos dados da FBCF anual da união é que, de acordo com Gobetti (2006), tem-se crescentemente contabilizado como "despesas de investimento da união" valores que efetivamente não foram gastos. A crescente importância dos "contingenciamentos" de despesas da União nos últimos anos - e, consequentemente, do procedimento contábil conhecido como "liquidação forçada” - vem gerando um viés nas despesas de investimento da União.

Para entender o argumento de Gobetti é importante ter em mente que a execução orçamentária brasileira consiste de duas etapas básicas, quais sejam: (i) o "empenho" de um dado recurso, que ocorre quando o governo se compromete a fazer determinada despesa e, portanto procede a compra/licitação dos bens e/ou serviços em questão (ou seja, quando tem início o processo de contratação do serviço); e (ii) a "liquidação" desse recurso, que consiste no reconhecimento por parte do governo de sua obrigação de pagar os fornecedores pelo total ou por partes dos bens e/ ou serviços comprados/licitados (de modo que se pode argumentar que é na liquidação que os efeitos econômicos do investimento são, de fato, observados).

Em segundo lugar, deve-se ter em mente que o governo tem frequentemente "contingenciado" recursos no começo do ano, a fim de garantir o cumprimento de suas metas fiscais anuais, liberando uma grande parte desses recursos no final do ano. Uma vez que é usualmente difícil para os administradores públicos gastar todo o montante "descontingenciado" no curto espaço de tempo que dispõem antes do fim do ano, tais recursos são liquidados mesmo sem terem sido gastos - e daí o termo "liquidação forçada". Ou seja, o procedimento da "liquidação forçada" nada mais é do que o "jeitinho" dado pelos administradores públicos para evitar que os empenhos liberados no final do ano sejam cancelados (como, por lei, deve ocorrer).

Tendo identificado o problema acima, Gobetti se propõe a mensurá-lo analisando duas séries de dados, i.e., (i) a obtida somando-se os investimentos efetivamente pagos em um dado ano e "os restos a pagar processados" desse ano; e (ii) a obtida pela exclusão dos "restos a pagar não processados" dos investimentos liquidados totais em um dado ano. Crucial para entender a racionalidade da sugestão de Gobetti, portanto, é a distinção entre "restos a pagar processados" (i.e., as despesas liquidadas e ainda não pagas, a despeito dos fornecedores dos bens/serviços já terem comprovado sua entrega) e os "restos a pagar não processados" (i.e., as despesas liquidadas forçadamente sem que os fornecedores/prestadores dos bens/ serviços em questão tenham comprovado a entrega/prestação).

Com efeito, ambas as séries de Gobetti seriam boas aproximações das despesas de investimento efetivamente realizadas em um dado ano se os "restos a pagar processados" de um dado ano fossem todos efetivamente pagos no ano seguinte. No entanto, Gobetti nota que as duas séries passaram a divergir de forma significativa a partir de 2001 quando os restos a pagar de vários anos se acumularam, de 
forma que os efeitos da liquidação forçada começaram a "transbordar" para os anos seguintes.

Gobetti, então, propôs um mapeamento da "origem" dos restos a pagar dos vários anos a partir de consultas no SIAFI. Com base nas considerações de Gobetti, propomos a seguinte metodologia de cálculo do investimento efetivo da União realizada em duas etapas:

$$
\begin{aligned}
& i_{F}^{* *}=i_{F}^{*}, t=1,2,3 \\
& i_{F}^{* *}=i_{F}^{*}-\left(i_{F L}-i_{F P}-i_{F R P P}\right), t=4 \\
& i_{F}=i_{F}^{* *}+\left(i_{E}-i_{C}\right) / 4
\end{aligned}
$$

em palavras, na primeira etapa (1), sempre que o dado se refere ao investimento do quarto trimestre $(t=4)$, foi feito o expurgo da "liquidação forçada" [i.e., o total das despesas de investimento "liquidadas" nesse trimestre $\left(i_{F L}\right)$ menos as despesas de investimento pagas $\left(i_{F P}\right)$ e as contabilizadas como "restos a pagar processados" $\left.\left(i_{F R P P}\right)\right]$. Na segunda etapa $(2)$, o estoque de restos a pagar de anos anteriores - calculado como a diferença entre o investimento efetuado $\left(i_{E}\right)$ e o investimento pelo primeiro critério de Gobetti ( $i_{C}$, que consiste na soma do investimento pago e dos restos a pagar processados) - foi distribuído entre os quatro trimestres de forma igual. Avaliando a validade das adaptações, comparamos nossos resultados (anualizado) com valores da base da SPE e de Gobetti (2006), apresentados na Tabela 3.

De posse da série ajustada acima, podemos então obter aproximações trimestrais para a formação bruta de capital fixo da união aplicando a sazonalidade trimestral da série ajustada (das "despesas de investimento" da União) às estimativas anuais ajustadas de Gobetti para a FBCF da União (Gobetti, 2006, p.45) e somá-la aos dados de estados e municípios para obter estimativas da FBCF da administração pública. O Gráfico 1 apresenta as principais diferenças entre a série original da SPE e o investimentos ajustados, ambos trimestralizados, bem como a FBCF pública.

Tabela 3: Comparação dos dados de Investimento ajustado - anual (em R $\$$ bilhões correntes)

\begin{tabular}{|c|c|c|c|}
\hline & SPE & Gobetti (2006) & Ajustado \\
\hline 1995 & 4,927 & 4,440 & 4,651 \\
\hline 1996 & 5,730 & 5,257 & 5,259 \\
\hline 1997 & 7,537 & 7,252 & 7,315 \\
\hline 1998 & 8,284 & 8,493 & 8,685 \\
\hline 1999 & 6,931 & 5,349 & 5,307 \\
\hline 2000 & 10,099 & 7,736 & 7,760 \\
\hline 2001 & 14,580 & 10,509 & 10,509 \\
\hline 2002 & 10,126 & 13,448 & 13,450 \\
\hline 2003 & 6,452 & 7,070 & 7,070 \\
\hline 2004 & 10,865 & 6,975 & 6,538 \\
\hline 2005 & 17,133 & 11,158 & 10,970 \\
\hline
\end{tabular}

Fonte: Elaborado pelos autores com base nos dados primários da SPE e Gobetti (2006). 
Gráfico 1: Despesas de Investimento da União (em $\mathrm{R} \$$ bilhões correntes)

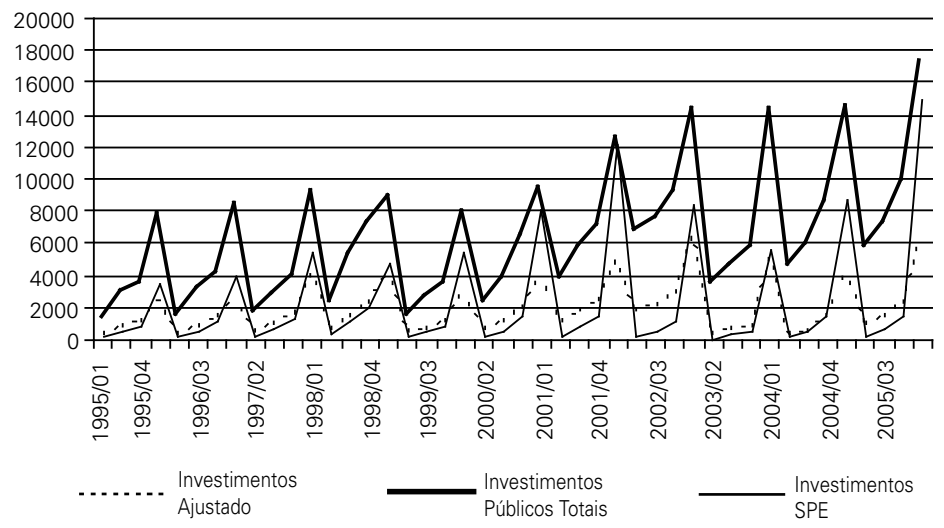

Fonte: Elaborado pelos autores com base nos dados primários da SPE e Gobetti (2006).

\section{REVISITANDO A "FUNÇÃO INVESTIMENTO PRIVADO" DO BRASIL APÓS O PLANO REAL COM DADOS TRIMESTRAIS}

As considerações acima deixam claro que existem vários conceitos possíveis para os "investimentos públicos". Aqui usaremos dois: (i) o conceito das contas nacionais (que inclui a FBCF dos governos federal, municipais e estaduais); e (ii) a série anterior adicionada da FBCF das empresas federais estatais. Os dois pares de séries foram deflacionados por uma média ponderada do "IPA-DI Máquinas e Equipamentos" (IPA-ME) com peso de $2 / 3$ e do "Índice Nacional de Custos de Construção" (INCC) com peso de 1/3 de modo a refletir a média da composição da FBCF (das contas nacionais anuais) no período.

Além dos logaritmos neperianos das medidas de investimento (dessazonalizadas pelo método X-12 multiplicativo), trabalhamos ainda com as seguintes variáveis: (i) a estimativa da carga tributária bruta trimestral total da economia medida como percentagem do PIB; (ii) uma proxy do preço relativo dos bens de capital dada pelo logaritmo neperiano da razão entre o "IPA-DI Bens de Produção" e o "IGP-DI", ambos da FGV (que não apresenta características sazonais); (iii) a série encadeada trimestral do logaritmo neperiano do PIB dessazonalizado "em volume" do IBGE.

Experimentamos ainda (i) as taxas Selic e "TJLP" medidas em termos reais; e (ii) os desembolsos do BNDES medidos como percentagem do PIB publicados pelo Banco Central. Os resultados obtidos com essas variáveis não foram bons. Enquanto as taxas de juros se mostraram robustamente estacionárias (e, portanto, inadequadas a análises de cointegração), os coeficientes obtidos com os desembolsos do BNDES apresentaram valores negativos em várias especificações. Esse fato talvez tenha a ver com o caráter excessivamente agregado do conceito de FBCF ou com complicações relacionadas a multicolinearidade (dada a alta correlação exis- 
tente entre os desembolsos do BNDES com o PIB e com a carga tributária) - mas mais pesquisas devem ser feitas antes de chegarmos a quaisquer conclusões.

\section{Testes de raiz unitária com e sem quebras estruturais}

Os resultados obtidos com a aplicação de testes convencionais de raízes unitárias parecem indicar que as séries de investimento privado, PIB e preço do capital são não-estacionárias, enquanto a carga tributária e o nível de investimento público aparentam ser estacionárias (Tabela 6, no Apêndice).

Há, entretanto, motivos para suspeitar da existência de quebras estruturais nas séries em questão e, portanto, que os resultados da Tabela 6 não devem ser tomados como definitivos (Perron, 1989). Com efeito, (i) os dados cobrem períodos de instabilidade que provocaram mudanças na política macroeconômica (Giambiagi, 2006); (ii) é teoricamente improvável que a tendência positiva apresentada pela série da carga tributária e a ausência de tendência apresentada pelas séries da FBCF pública real da economia sejam características permanentes; (iii) grande parte do antigo parque produtivo estatal brasileiro foi privatizado na segunda metade dos anos 1990; e (iv) é notório que a carga tributária dá um salto a partir da crise de 1999 e outro em 2002 (Giambiagi, 2006), enquanto as séries da FBCF pública real da economia caem antes da crise de 1999 e no começo do governo Lula. Assim sendo, a aplicação de testes de raiz unitária que levam explicitamente em consideração a possibilidade de quebras estruturais é crucial (Tabela 7, no Apêndice).

De fato, são pelo menos três os problemas com os testes de raízes unitárias tradicionais na presença de quebras estruturais. Em primeiro lugar, a presença de uma quebra estrutural em uma série tendência-estacionária faz com que seja difícil rejeitar a hipótese de que a série em questão é um caminho aleatório em torno de uma tendência (Perron, 1989). Em segundo lugar, a presença de observações aberrantes em um caminho aleatório em torno de uma tendência pode fazer com que se rejeite a hipótese de que a série em questão é não-estacionária (Franses e Haldrup, 1994). Em terceiro lugar, a presença de quebras estruturais em caminhos aleatórios "puros" pode tornar difícil rejeitar a hipótese que a série em questão é tendência-estacionária (Leybourne et al., 1998).

A fim de investigar a possibilidade dos resultados que obtivemos na Tabela 6 para as séries do PIB e do investimento privado terem sido causados pela presença de quebras estruturais, aplicamos os testes de Zivot-Andrews (1992) e Lanne et al. (2003). Ambos os testes são variações do conhecido teste de Perron (1989), com a diferença de que determinam o "timing" da quebra estrutural endogenamente 7 . A aplicação desses testes nos permite, assim, obter insights valiosos sobre os pontos

\footnotetext{
${ }^{7}$ Os testes de Lanne et al. e Zivot e Andrews apresentam diferenças, entretanto. A hipótese nula do teste de Zivot e Andrews é de um random walk com tendência e sem quebra estrutural, enquanto a hipótese nula do teste de Lanne et al. é de um random walk com tendência e quebra estrutural.
} 
de quebra mais prováveis e testar a hipótese nula de raiz unitária com tendência nesses casos (Tabela 7).

Analisando a Tabela 7, percebe-se que os resultados obtidos com os testes de Zivot e Andrews e de Lanne et al. são frequentemente parecidos e, grosso modo, compatíveis com a interpretação "histórica" mencionada acima. Esses resultados reforçam as conclusões apresentadas na Tabela 6 no caso das variáveis PIB e PK, mas não sobre as conclusões obtidas com as variáveis de investimento. A fim de verificar a possibilidade da evidência de estacionaridade que obtivemos para as variáveis de investimento ter sido "espúria" no sentido de Franses e Haldrup (1994), decidimos então aplicar o teste sugerido por esses autores ${ }^{8}$. Conforme se observa na Tabela 7, os resultados para as séries de investimento privado reforçam nossa conclusão anterior de que elas não são estacionárias (considerando os choques "aberrantes"). Ademais, as evidências não nos permitem afastar a hipótese de que os investimentos públicos (notadamente a série que inclui os investimentos das estatais federais) são não-estacionários.

A fim de investigar a hipótese de que a carga tributária medida como percentagem do PIB é um caminho aleatório que sofreu "choques" positivos no meio de 1999 e no começo de 2003, rodamos o teste proposto por Franses e Haldrup também nessa variável. Conforme se observa na Tabela 7, os resultados obtidos reforçam a tese de que a variável carga tributária tem, de fato, uma raiz unitária.

\section{Testes de Cointegração com e sem Quebras Estruturais}

$\mathrm{Na}$ presença de quebras estruturais nas relações de cointegração, "rejeições espúrias" da hipótese de "não cointegração" nos testes convencionais (e.g., Engle e Granger, 1987; Johansen, 1991; e Banerjee et al., 1986) são comuns (Lutkepohl e Kratzig, 2004). Por outro lado, a mera existência de quebras estruturais nas séries individuais não implica necessariamente a existência de quebras estruturais nas relações de cointegração que porventura existam entre essas séries. Dito de outro modo, é perfeitamente possível que duas variáveis passem a se comportar de modo diferente em um dado momento do tempo sem que a relação de longo prazo entre ela tenha mudado.

Os dois conceitos (i.e., quebras estruturais nas séries individuais e nas relações de cointegração) estão relacionados, entretanto. Com efeito, pesquisas recentes (Leybourne e Newbold, 2003; Cook, 2004) apontam que a presença de uma quebra estrutural nas séries individuais frequentemente leva a "rejeições espúrias" da hipótese de "não-cointegração" nos testes de cointegração convencionais mencionados acima e no teste de cointegração com quebra estrutural de Gregory e Hansen (1996).

Felizmente, essa literatura parece indicar que os diferentes testes de cointegra-

\footnotetext{
${ }^{8} \mathrm{O}$ teste de Franses e Haldrup é uma variação do teste ADF com dummies de pulso (predefinidas) nas datas em que são observadas as observações aberrantes (Patterson, 2000).
} 
ção são afetados de forma diferente por diferentes quebras (Leybourne e Newbold, 2003; Cook, 2004) - de modo a fornecer alguma base para a avaliação dos resultados desses testes. Com efeito, a mensagem básica dessa literatura parece ser a de que análises de cointegração na presença de quebras estruturais devem se basear em um julgamento dos resultados de um número relativamente grande de testes de cointegração com e sem quebras estruturais e de testes de constância paramétrica dos modelos vetoriais de correção de erros.

Assim sendo, começamos por aplicar testes de cointegração "uniequacionais" (Tabela 4). A despeito dos conhecidos problemas desses testes no caso da existência de mais de um vetor de cointegração, os resultados obtidos com a aplicação do teste de Gregory e Hansen são particularmente importantes por nos darem uma ideia do "timing" da quebra estrutural. Além disso, os resultados do teste de Engle e Granger também devem ser considerados, uma vez que esse se mostrou o mais eficiente na presença de quebras estruturais nas séries envolvidas (de acordo com Leybourne e Newbold, 2003).

Tabela 4: Resultados dos testes de cointegração "uniequacionais"

\begin{tabular}{|c|c|c|c|}
\hline Teste & Especificação & Resultado & Relação de Cointegração \\
\hline Engle e Granger & Priv $=f(p i b, c t, p k, p u b)$ & $\begin{array}{l}\text {-4.53 (rejeita a hipótese } \\
\text { nula a 10\% mas não a 5\%) }\end{array}$ & $\begin{aligned} \text { Priv }= & -1.87+1.92 p i b-.81 p k \\
& -1.09 c t-.14 p u b\end{aligned}$ \\
\hline Engle e Granger & Priv $=f(p i b, c t, p k)$ & $\begin{array}{c}\text {-3.93 (rejeita a hipótese } \\
\text { nula a 15\% mas não a 10\%) }\end{array}$ & $\begin{array}{c}\text { Priv }=-2.15+1.74 p i b-.62 p k \\
-0.95 c t\end{array}$ \\
\hline Engle e Granger & Priv $=f(p i b, p k, p u b)$ & $-4.84^{*}$ & $\begin{array}{c}\text { Priv }=-.025+1.6 p i b-.96 p k- \\
.13 p u b\end{array}$ \\
\hline Engle e Granger & Priv $=f(p i b, p k)$ & $-4.15^{*}$ & Priv $=-.52+1.47 p i b-.76 p k$ \\
\hline Gregory-Hansen & Priv $=f(p i b, c t, p k, p u b)$ & $\begin{array}{c}-6.61 * \text { Quebra estimada } \\
\text { em 2001:4 }\end{array}$ & $\begin{array}{c}\text { Priv }=-3.62+1.78 p i b-.33 p k \\
-0.61 c t-.1 p u b \text { (antes de } 2001: 4) \text { e } \\
\text { Priv }=-6.81+2.05 p i b+.06 p k \\
-1.41 c t+.04 p u b \text { (depois de } \\
2001: 4)\end{array}$ \\
\hline Gregory-Hansen & Priv $=f(p i b, c t, p k)$ & $\begin{array}{c}-6.15^{*} \text {. Quebra estimada } \\
\text { em 2001:4 }\end{array}$ & $\begin{array}{c}\text { Priv }=-3.81+1.65 p i b-.21 p k \\
-0.03 c t \text { (antes de 2001:4) e } \\
\text { Priv }=-6.15+2.08 p i b-1.18 p k \\
-0.0125 c t \text { (depois de 2001:4) }\end{array}$ \\
\hline Gregory-Hansen & Priv $=f(p i b, p k, p u b)$ & $\begin{array}{c}-6.79 * * \text {. Quebra estimada } \\
\text { em 2001:4 }\end{array}$ & $\begin{array}{c}\text { Priv }=-2.7+1.63 p i b-.41 p k \\
-0.08 p u b \text { (antes de } 2001: 4 \text { ) e } \\
\text { Priv }=-5.25+1.6 p i b+.115 p k+ \\
\text { 0.15pub (depois de 2001:4) }\end{array}$ \\
\hline Gregory-Hansen & Priv $=f(p i b, p k)$ & $\begin{array}{c}-6.09 * * \text {. Quebra estimada } \\
\text { em 2001:4 }\end{array}$ & $\begin{array}{c}\text { Priv }=-3.9+1.66 p i b-.21 p k \\
\text { (até2001:4) e Priv }=-5.11+1.64 \text { pib } \\
+.04 p k+(\text { depois de } 2001: 4)\end{array}$ \\
\hline
\end{tabular}

${ }^{*}\left({ }^{*}\right)$ indica rejeição da hipótese nula de não-cointegração ao nível de significância de 5\% (1\%). Priv e Pub denotam, respectivamente, as séries de FBCF privada (excluindo estatais) e pública (incluindo estatais).

Nota: Os testes acima supõem que a relação de cointegração é linear e sem tendência determinística.

Fonte: Elaborado pelos autores.

Concluímos, a partir da Tabela 4, que os testes uniequacionais indicam fortemente a existência de um vetor de cointegração entre as séries apresentadas acima, 
notadamente se tiver havido, de fato, uma quebra estrutural em 2001:4, como parecem indicar os testes de Zivot e Andrews e de Gregory e Hansen. A Tabela 4 apresenta também fortes evidências em favor de elevadas elasticidade-produto (positiva) e semielasticidade-carga tributária (negativa) do investimento privado. A evidência acerca das elasticidades preço do capital e investimento público é bem menos contundente, entretanto.

As estimativas pontuais obtidas nos modelos de regressão simples não são confiáveis no caso de haver mais de um vetor de cointegração (por captarem combinações lineares dos vários vetores de cointegração), entretanto. A fim de controlar pela possibilidade de múltiplos vetores de cointegração efetuamos o procedimento de cointegração vetorial de Johansen (1991), obtendo resultados em linha com os obtidos anteriormente (ver Tabela 5) ainda que com níveis de significância acima dos convencionais, por conta das quebras estruturais que não são consideradas nessa abordagem (Lutkepohl e Kratzig, 2004, cap. 3).

Tabela 5: Resultados dos testes de cointegração de Johansen convencionais ${ }^{9}$

\begin{tabular}{|c|c|c|}
\hline $\begin{array}{l}\text { VAR de partida (c/ termo } \\
\text { constante e s/tendência) }\end{array}$ & Resultado & Relação de Cointegração (VECM) \\
\hline $\begin{array}{l}\text { VAR ordem } 2 \text { e com resíduos aparente- } \\
\text { mente normais, homocedásticos e não cor- } \\
\text { relacionados e composto por priv, pib, pk, ct } \\
\text { e pub. Testes de quebra estrutural de Chow } \\
\text { apontam quebras em 2000:4 e 2001:2 }\end{array}$ & $\begin{array}{l}\text { Teste do traço re- } \\
\text { jeita a não-cointe- } \\
\text { gração a } 21.3 \%\end{array}$ & $\begin{array}{l}\text { VECM ordem } 1 \text { com resíduos aparentemente } \\
\text { normais, homocedásticos e independentes e } \\
\text { vetor de cointegração: } \\
\text { Priv=-1.68+2.22pib-1.18pk -0.91ct-.16pub } \\
\text { Modelo estável }\end{array}$ \\
\hline $\begin{array}{l}\text { VAR ordem } 2 \text { com resíduos aparente- } \\
\text { mente normais, homocedásticos e } \\
\text { independentes composto por priv, pib, } \\
\text { pk e ct. Testes de quebra estrutural de } \\
\text { Chow apontam várias quebras no período } \\
\text { 1999:1-2001:4 }\end{array}$ & $\begin{array}{l}\text { Teste do traço re- } \\
\text { jeita a não-cointe- } \\
\text { gração a } 16.5 \%\end{array}$ & $\begin{array}{l}\text { VECM ordem } 1 \text { com resíduos aparentemente } \\
\text { normais, homocedásticos e independentes e } \\
\text { vetor de cointegração: } \\
\text { Priv }=4.72+2.72 p i b-1.01 p k-2.31 \mathrm{ct} \text {. } \\
\text { O teste de quebra estrutural de Chow aponta } \\
\text { uma quebra em } 2001: 3\end{array}$ \\
\hline $\begin{array}{l}\text { VAR ordem } 2 \text { "congruente" ( i.e., aparente- } \\
\text { mente estável e com resíduos aparente- } \\
\text { mente normais, homocedásticos e indepen- } \\
\text { dentes), composto por priv, pib, pk e pub }\end{array}$ & $\begin{array}{l}\text { Teste do traço } \\
\text { rejeita a não-coin- } \\
\text { tegração a } 25 \%\end{array}$ & $\begin{array}{l}\text { VECM ordem } 1 \text { congruente e com vetor } \\
\text { de cointegração: } \\
\text { Priv }=.14+1.95 p i b-1.3 p k-0.15 p u b\end{array}$ \\
\hline $\begin{array}{l}\text { VAR de ordem } 2 \text { com resíduos aparente- } \\
\text { mente normais, e não correlacionados } \\
\text { (mas com evidência de heterocedasticid- } \\
\text { ade) composto por priv, pib e pk. Testes } \\
\text { de quebra estrutural de Chow apontam } \\
\text { quebras em 1998:3, 2001:3 e 2003:2 }\end{array}$ & $\begin{array}{l}\text { Teste do traço re- } \\
\text { jeita a não-cointe- } \\
\text { gração a } 21.5 \%\end{array}$ & $\begin{array}{l}\text { VECM ordem } 1 \text { com resíduos aparentemente } \\
\text { normais e não correlacionados (mas com } \\
\text { alguma de heterocedasticidade) e vetor de } \\
\text { cointegração: } \\
\text { Priv }=0.76+2.09 \text { pib }-1.37 p k \\
\text { Modelo estável. }\end{array}$ \\
\hline
\end{tabular}

Fonte: Elaborado pelos autores.

\footnotetext{
${ }^{9}$ Ordem dos VARs foi escolhida de modo a minimizar critérios de perda (Akaike, Schwartz, Hannah-Quinn, Mínimo Erro de Previsão) e garantir que nenhuma defasagem significante (no teste de Wald) fosse excluída. A despeito de várias especificações terem se mostrado incongruentes, variáveis dummy não foram incluídas para não viesar os resultados. Os testes de normalidade, heterocedasticidade e não autocorrelação utilizados foram, respectivamente, Jarque-Bera, White e Portmanteu e LM. Os testes de Chow foram realizados no programa Jmulti versão 4.15. Os demais no programa E-Views versão 4.1.
} 
Tomados em conjunto, os resultados acima parecem compatíveis com uma elasticidade-PIB do investimento próxima de 2 e semielasticidade carga tributária do investimento próxima de menos um. Infelizmente, não temos como ir além da mera inspeção estimativas pontuais, uma vez que (i) a aplicação dos testes t e F não é válida no caso de relações de cointegração ${ }^{10}$; e (ii) não há evidência de que os valores críticos dos testes recomendados pela literatura nesse caso (i.e., os baseados em modelos de mínimos quadrados dinâmicos ou "totalmente modificados" no caso de modelos uniequacionais e no procedimento de máxima verossimilhança no caso de modelos vetoriais) sejam robustos a existência de quebras estruturais.

\section{ALGUMAS CONCLUSÕES PARCIAIS E O ESBOÇO DE UMA AGENDA DE PESQUISA}

As duas conclusões mais importantes do estudo são que: (i) a elasticidade-produto do investimento privado brasileiro parece ser elevada, sendo maior que 1,5 em praticamente todos os modelos estimados nesse texto e ficando próxima de 2 em vários desses últimos; e (ii) a semielasticidade-carga tributária do investimento privado brasileiro também parece ser significativa, ficando próxima de menos um em grande parte dos modelos estimados. Enquanto a primeira conclusão apenas reafirma os resultados obtidos em estudos anteriores, a segunda conclusão é, até onde sabemos, inédita e implica que as políticas governamentais redistributivas ora em curso têm tido um custo relativamente pequeno em termos de diminuição do investimento. Com efeito, o fato da semielasticidade carga tributária do investimento ser próxima de menos um implica que aumentos de $1 \%$ do PIB (R $\$ 23,2$ bilhões em 2006) na carga tributária bruta estão associados a diminuições de $1 \%$ no investimento privado (cerca de R \$3,2 bilhões em 2006).

Naturalmente, nossas conclusões foram obtidas com dados aproximados e, portanto, devem ser vistas com cuidado. Por outro lado, não é comum que dados ruins gerem conclusões robustas a diferentes especificações econométricas e em linha com a teoria econômica, de modo que nossos resultados nos parecem sugestivos o suficiente para incentivar outros pesquisadores a reavaliarem nossas hipóteses de outras maneiras e com outros dados.

Finalmente, notamos que a base de dados trimestral apresentada possibilita inúmeros experimentos mais detalhados que os apresentados nesse artigo. Não há nenhum motivo para acharmos que os diferentes componentes da carga tributária bruta afetam o investimento privado da mesma forma, ou que diferentes conceitos dessa última variável sejam descritos pelas mesmas especificações econométricas. Tal fato evidencia a existência de uma linha de pesquisa de considerável importância para o esclarecimento dos determinantes da dinâmica da economia brasileira e dos trade-offs à disposição da sociedade.

${ }^{10}$ Ver Patterson (2000, cap. 8). 


\section{REFERÊNCIAS BIBLIOGRÁFICAS}

BANERJEE, A., DOLADO, J., HENDRY, D. and SMITH, G. (1986). "Exploring equilibrium relationships in econometrics through static models: some Monte Carlo evidence". Oxford Bulletin of Economics and Statistics, 48, 253-77.

CAVALCANTI, M.A.F.H., KAI, H. e CARVALHO, L. (2002) Principais Características do Modelo Macroeconômico do IPEA. Seminários DIMAC - IPEA (seminário apresentado em 26 de junho de 2002).

COOK, S. (2004). "Spurious rejection by cointegration tests incorporating structural change in the cointegrating relationship". Applied Economics Letters 11, 879-884.

CRUZ, B. O. e TEIXEIRA, J. R. (1999). "The impact of public investment on private investment in Brazil, 1947-1990”, Cepal Review, 67: 71-80.

DELFIM NETTO, A. (1973). "Prefácio". Em LANGONI, C.G (1973). Distribuição de Renda e Desenvolvimento Econômico no Brasil. Rio de Janeiro: Expressão e Cultura.

DOS SANTOS, C.H e COSTA, F.R. (2007). Uma Metodologia Simplificada de Estimação da Carga Tributaria Brasileira Trimestral. IPEA. Texto para Discussão em fase de publicação. Brasília-DF.

DICKEY, D. e FULLER, W. (1981). "Likelihood ratio statistics for autoregressive time series with a unit root. Econometrica. Vol.49: 1022-1057.

DICKEY, D. e PANTULA, S. (1987). "Determining the order of differencing in autoregressive processes". Journal of Business and Economic Statistics. Vol.5: 455-461.

ENGLE, R. F. and GRANGER, C. W. J. (1987) "Cointegration and error correction: representation, estimation and testing". Econometrica, 55: 251-276.

FELDSTEIN, M. e HORIOKA, C. (1980). "Domestic saving and international capital flows", The Economic Journal, v. 90, n. 358, 314-329.

FRANSES, P.H. e HALDRUP, N. (1994). "The effects of additive outliers on tests for unit roots and cointegration”. Journal of Business and Economic Statistics. Vol.12. 471-478.

GIAMBIAGI, F. (2006). A política fiscal do governo Lula em perspectiva histórica: qual é o limite para o Aumento do gasto público? IPEA. Texto para Discussão n. 1169. Rio de Janeiro-RJ.

GOBETTI, S. (2006). Estimativa dos Investimentos Públicos: Um Novo Modelo de Analise de Execução Orçamentária Aplicado às Contas Nacionais. XI Prêmio do Tesouro Nacional. Secretaria do Tesouro Nacional.

GREGORY e HANSEN (1996). "Residual-based tests for cointegration in models with regime shifts". Journal of Econometrics. 70, 99-126.

HOGLUND, R. e OSTERMARK, R. (2003). "Size and power of cointegration tests under structural breaks and heteroskedastic noise". Statistical Papers. 44. 1-22.

HYLLEBERG, S.;ENGLE, R; GRANGER, C; e YOO, B. (1990). "Seasonal integration and cointegration”. Journal of Econometrics. Vol.44. 215-228.

JOHANSEN, S. (1988). "Statistical analysis of cointegrating vectors". Journal of Economic Dynamics and Control 12, 231-254.

(1991). "Estimation and hypothesis testing of cointegration vectors in Gaussian vector autoregressive models". Econometrica 59: 1551-1580.

KWIATKOWSKI, D.; PHILLIPS, P.; SCHMIDT; P; e SHIN, Y. (1992). "Testing the null hypothesis of stationarity against the alternative of a unit root", Journal of Econometrics, Vol.54: 159-178.

LANNE, M., LÜTKEPOHL, H., SAIKKONEN, P. (2003). "Test procedures for unit roots in time series with level shifts at unknown time", Oxford Bulletin of Economics and Statistics. 65: 91115 .

LEYBOURNE, S. J., MILLS, T. C. and NEWBOLD, P. (1998). "Spurious rejections by Dickey-Fuller tests in the presence of a break under the null”, Journal of Econometrics, 87: 191-203.

LEYBOURNE, S. e NEWBOLD, P. (2003). "Spurious rejections by cointegration tests induced by structural breaks”, Applied Economics. 35: 1117-1121.

MELO, G. M. e RODRIGUES Jr., W. (1998). Determinantes do investimento privado no Brasil: 19701995. IPEA. Texto para Discussão n.605. Brasília-DF. 
MUINHOS, M.K. e ALVES, S.A.L. (2003). Medium Size Macroeconomic Model for the Brazilian Economy. Banco Central do Brasil. Working Paper Series, 64. Brasília-DF.

NOH e KIM (2003). "Behaviour of cointegration tests in the presence of structural breaks in variance". Applied Economics Letters. 10: 999-1002.

PATTERSON, K. (2000). Introduction to Applied Econometrics: A Times Series Approach. Nova York: St. Martin's Press.

PASTORE. A. C e PINOTTI, M. C. (2006). Política Macroeconômica, Choque Externo e Crescimento. Instituto Nacional de Altos Estudos. Estudos e Pesquisas n.141. Rio de Janeiro. RJ.

PEREIRA, R. M. (2001). "Investment and uncertainty in a quadratic adjustment model", Revista Brasileira de Economia. 55(2): 283-311.

PERRON, P. (1989). "The great crash, the oil price shock and the unit root hypothesis", Econometrica 57: 1361-1401.

REIS, E., CAVALCANTI, M. A., CASTRO, A. R., ROSSI, Jr. J. L., EMERSON, R. A. e HERNANDES, B. M. (1999). Model for Projections and Simulations of the Brazilian Economy. IPEA. Texto para discussão n.619. Rio de Janeiro-RJ.

RIBEIRO, M.B. e TEIXEIRA, J. R. (2001). “An economic analysis of private-sector investment in Brasil", Cepal Review, 74:153-166.

ROCHA, C.H. e TEIXEIRA, J R. (1996). “Complementaridade versus substituição entre investimento público e privado na economia brasileira: 1965-90”, Revista Brasileira de Economia, 50(3): 378283. jul./set.

SACHSIDA, A. (1999). Testes de Exogeneidade sobre a Correlação Poupança Doméstica e Investimento. IPEA. Texto para Discussão n.659. Brasília-DF.

SACHSIDA, A. e ABI-RAMIA, M.C. (2000). "The Feldstein-Horioka puzzle revisited”, Economics Letters, v. 68: 85-88.

SACHSIDA, A. e MENDONÇA, M.J. (2006). "Domestic saving and investment revised: can the Feldstein-Horioka equation be used for policy analysis? "Texto para Discussão n.1158. BrasíliaDF.

SOARES, S. 2006. Distribuição de Renda no Brasil de 1976 a 2004 com ênfase no período 2001-2004. IPEA. Texto para discussão n.1166. Brasília-DF.

VELLOSO, R. (2006). Escancarando o Problema Fiscal: é preciso controlar o gasto não-financeiro obrigatório da União. Instituto Nacional de Altos Estudos. Estudos e Pesquisas n.159. Rio de Janeiro. RJ.

ZIVOT, E. e ANDREWS, D.W.K. (1992). "Further evidence on the great crash, the oil price shock and the unit root hypothesis", Journal of Business and Economic Statistics 10: 251-270. 


\section{Apêndice econométrico}

Tabela 6: Testes de raízes unitárias convencionais

\begin{tabular}{|c|c|c|c|c|}
\hline Séries/ Testes & ADFt Nível & ADFt $1^{\text {a }}$ Dif & $\begin{array}{l}\text { KPSS } \\
\text { Nível }^{* *}\end{array}$ & Decisão Provisória \\
\hline $\begin{array}{l}\text { Inv Privado (Contas } \\
\text { Nacionais) c/ constante }\end{array}$ & -1.29 & $-6.44 * *$ & $0.509^{*}$ & \multirow{4}{*}{$I(1)$} \\
\hline $\begin{array}{l}\text { Inv Priv (Contas Nac) c/ } \\
\text { tendência e cte }\end{array}$ & -2.66 & $A D$ & 0.10 & \\
\hline $\begin{array}{l}\text { Inv Priv (excluindo estatais } \\
\text { federais) c/ cte }\end{array}$ & -1.39 & $-6.76^{* *}$ & $0.508^{*}$ & \\
\hline $\begin{array}{l}\text { Inv Priv (excluindo est. } \\
\text { federais) c/ tend e cte }\end{array}$ & -2.79 & $A D$ & 0.10 & \\
\hline $\begin{array}{l}\text { Inv Público (Contas Nacio- } \\
\text { nais) c/ constante }\end{array}$ & -2.76 & $A D$ & 0.22 & \multirow{4}{*}{$\begin{array}{c}\mathrm{I}(0) \text { no modelo } \mathrm{c} / \\
\text { constante e I(1) no } \\
\text { modelo } \mathrm{c} / \text { tendência e } \\
\text { constante }\end{array}$} \\
\hline $\begin{array}{l}\text { Inv Pub (Contas Nacionais) } \\
\text { c/ tend. e cte }\end{array}$ & -2.94 & $A D$ & 0.09 & \\
\hline $\begin{array}{l}\text { Inv Pub (incluindo estatais } \\
\text { federais) c/ cte }\end{array}$ & -2.54 & $A D$ & 0.18 & \\
\hline $\begin{array}{l}\text { Inv Pub (incluindo est. } \\
\text { federais) c/ tend e cte }\end{array}$ & -2.69 & $A D$ & 0.05 & \\
\hline PIB c/ tendência e cte & -3.06 & $A D$ & 0.10 & $I(1)^{D}$ \\
\hline Carga Trib. c/ cte & 0.016 & $-13.24^{* *}$ & $0.87^{* *}$ & \multirow{2}{*}{$\begin{array}{c}\text { I(1) no modelo c/ cte e } \\
\text { I(0) no modelo c/ tend } \\
\text { e cte. }\end{array}$} \\
\hline Carga Trib. c/ tend. e cte & $-5.25 * *$ & $A D$ & 0.09 & \\
\hline Preço K c/cte & -0.27 & $-4.39 * *$ & $0.76^{* *}$ & \multirow{2}{*}{$\mid(1)$} \\
\hline Preço K c/tend e cte & -2.5 & $A D$ & $0.15^{*}$ & \\
\hline
\end{tabular}

* A sigla KPSS se refere às iniciais dos autores que propuseram o teste de raiz unitária em que a hipótese nula é a de estacionariedade da série. Ver Kwiatkowski, Phillips, Schmidt e Shin (1992).

** Indica rejeição da hipótese nula ao nível de significância de 5\%(1\%). AD indica aplicação desnecessária à luz da inspeção gráfica das séries e/ou dos demais resultados.

Fonte: Elaborado pelos autores. 
Tabela 7: Testes de raízes unitárias c/ quebras estruturais*

\begin{tabular}{|c|c|c|c|}
\hline $\begin{array}{l}\text { Séries/ } \\
\text { Testes }\end{array}$ & $\begin{array}{l}\text { Z\&A (Hipotése Nula: } \\
\text { Caminho Aleatório } \\
\text { com Tendência) }\end{array}$ & $\begin{array}{l}\text { Lanne et al. } \\
\text { (Hipotése Nula: } \\
\text { Caminho Aleatório } \\
\text { com Tendência) }\end{array}$ & F\&H (Hipotése Nula: Raiz Unitária) \\
\hline $\begin{array}{l}\text { Inv Privado } \\
\text { (Cont. Nac) }\end{array}$ & $\begin{array}{l}-4.95 \text { (1995:1-2006:3 c/ } \\
\text { quebra est. em 2001:4) }\end{array}$ & $\begin{array}{l}-1.86(1995: 1-2006: 3 \mathrm{c} / \\
\text { quebra est. em 2000:1); } \\
-2.94^{* *}\end{array}$ & $\begin{array}{l}-2.29 \text { (1998:1-2006:3, modelo c/ } \\
\text { tendência e outliers em 2000:1, 2001:4 } \\
\text { e 2005:2) }\end{array}$ \\
\hline $\begin{array}{l}\text { Inv. Privado } \\
\text { (exclusive } \\
\text { estatais } \\
\text { federais) }\end{array}$ & $\begin{array}{c}-5.83 * *(1995: 1-2006: 3 \\
\text { c/ quebra est. em } \\
2001: 4)\end{array}$ & $\begin{array}{l}-1.92 \text { (1995:1-2006:3 c/ } \\
\text { quebra est. em 2000:2) }\end{array}$ & $\begin{array}{l}-2.39 \text { (1998:1-2006:3, modelo c/ } \\
\text { tendência e outliers em 2000:1, 2001:4 } \\
\text { e 2005:2) }\end{array}$ \\
\hline $\begin{array}{l}\text { Inv Público } \\
\text { (Cont. Nac) }\end{array}$ & $\begin{array}{c}-4.79(1995: 1-2006: 3 \mathrm{cl} \\
\text { quebra est. em 2003:1) }\end{array}$ & $\begin{array}{l}-1.57 \text { (1995:1-2006:3 c/ } \\
\text { quebra est. em 2002:1) }\end{array}$ & $\begin{array}{l}-3.91 * \text { (1999:1-2006:3, modelo s/ tend. } \\
\text { e c/ outliers em 2002:1 e 2003:1); } \\
-3.85 * \text { (1999:1-2006:3, modelo c/ tend. } \\
\text { e outliers em 2002:1 e 2003:1) }\end{array}$ \\
\hline $\begin{array}{l}\text { Inv. Público } \\
\text { (inclusive } \\
\text { estatais } \\
\text { federais) }\end{array}$ & $\begin{array}{l}-3.65 \text { (1995:1-2006:3 c/ } \\
\text { quebra est. em 1998:4) }\end{array}$ & $\begin{array}{l}-2.58 \text { (1995:1-2006:3 c/ } \\
\text { quebra est. em 1998:4) }\end{array}$ & $\begin{array}{l}-3.297 \text { (1999:1-2006:3, modelo s/ tend. e } \\
\text { c/ outliers em 2002:1 e 2003:1); } \\
\text {-3.372 (1999:1-2006:3, modelo c/ tend. e } \\
\text { outliers em 2002:1 e 2003:1) }\end{array}$ \\
\hline PIB & $\begin{array}{l}-4.2 \text { (1995:1-2006:3 c/ } \\
\text { quebra est. em 1998:1) }\end{array}$ & $\begin{array}{l}-0.45(1995: 1-2006: 3 \mathrm{c} / \\
\text { quebra est. em 1998:2) }\end{array}$ & $A D$ \\
\hline Preço do K & $\begin{array}{l}\text {-3.5 (1995:1-2006:3 c/ } \\
\text { quebra est. em 1999:1) }\end{array}$ & $\begin{array}{l}-0.21 \text { (1995:1-2006:3 c/ } \\
\text { quebra est. em 1999:1) }\end{array}$ & $A D$ \\
\hline $\begin{array}{c}\text { Carga } \\
\text { Tributária }\end{array}$ & $A D$ & $A D$ & $\begin{array}{l}-0.75 \text { e -2.61 (1995:1-2006:3, } \\
\text { especificações s/ e c/ tendência e c/ } \\
\text { outliers em 1999:2 e 2003:1. }\end{array}$ \\
\hline
\end{tabular}

*Em todos os testes de Zivot e Andrews assumiu-se que tanto a constante quanto o intercepto das séries foram alterados pela quebra e utilizou-se o critério de Akaike para selecionar a especificação precisa da equação de teste. Em todos os testes de Lanne et al. a "função quebra" utilizada foi a exponencial (ver Lutkepohl e Kratzig, 2004) e utilizou-se o critério de Akaike para selecionar a especificação da equação.

* Indica rejeição da hipótese nula ao nível de significância de 5\% (1\%). AD indica aplicação desnecessária em nosso julgamento.

Fonte: Elaborado pelos autores. 